\title{
Two New Ultra-Faint Star Clusters in the Milky Way Halo
}

\author{
Dongwon Kim \\ Research School of Astronomy and Astrophysics, The Australian National University, Mt \\ Stromlo Observatory, via Cotter Rd, Weston, ACT 2611, Australia \\ email: dongwon.kim@anu.edu.au
}

\begin{abstract}
Kim 1 \& 2 are two new star clusters discovered in the Stromlo Missing Satellite Survey. Kim 1, located at a heliocentric distance of $19.8 \pm 0.9 \mathrm{kpc}$, features an extremely low total luminosity $\left(M_{V}=0.3 \pm 0.5 \mathrm{mag}\right)$ and low star concentration. Together with the large ellipticity $(\epsilon=0.42 \pm 0.10)$ and irregular isophotes, these properties suggest that Kim 1 is an intermediate mass star cluster being stripped by the Galactic tidal field. Kim 2 is a rare ultrafaint outer halo globular cluster located at a heliocentric distance of $104.7 \pm 4.1 \mathrm{kpc}$. The cluster exhibits evidence of significant mass loss such as extra-tidal stars and mass-segregation. Kim 2 is likely to follow an orbit confined to the peripheral region of the Galactic halo, and/or to have formed in a dwarf galaxy that was later accreted into the Galactic halo.
\end{abstract}

Keywords. Galaxy, halo, globular clusters, dwarf galaxies.

\section{Discovery of Kim $1 \& 2$}

Kim 1 \& 2 are ultra-faint stellar systems recently discovered by Kim \& Jerjen (2015a) and Kim et al. (2015a) in the Stromlo Missing Satellite (SMS) survey (PI: Helmut Jerjen) (see also Kim et al. 2015b and Kim \& Jerjen 2015b). Kim 1 was first detected in the preexisting Sloan Digital Sky Survey Data Release 10 (Ahn et al. 2014) and Kim 2 in an independent 500 sqr degree survey using the Dark Energy Camera (DECam) at the $4 \mathrm{~m}$ Blanco telescope at CTIO as a part of the SMS survey. Their true identities were confirmed by deep follow-up imaging using DECam and the Gemini-South 8-m telescope respectively (Fig. 1). Both objects are located in the Vast Polar Structure (Pawlowski et al. 2015).

\section{Properties of the Star Clusters and Implications}

Kim 1 features an old $\left(12_{-3.0}^{+1.5} \mathrm{Gyr}\right)$ and metal-poor $\left([\mathrm{Fe} / \mathrm{H}]=-1.7_{-0.2}^{+0.5}\right)$ stellar population at a heliocentric distance of $19.8 \pm 0.9 \mathrm{kpc}$. Its small physical size $\left(r_{h}=6.9 \pm 0.6 \mathrm{pc}\right)$ and the extremely low luminosity $\left(M_{V}=0.3 \pm 0.5\right)$ are comparable to those of the faintest known star clusters such as Segue 3, Koposov 1 \& 2, and Muñoz 1 (Fadely et al. 2011; Koposov et al. 2007; Muñoz et al. 2012). However, Kim 1 exhibits an usually low star concentration, large ellipticity $(\epsilon=0.42 \pm 0.10)$ and irregular outer isophotes, and is lacking a well defined center. The observed properties suggest that Kim 1 is most likely an intermediate mass star cluster being stripped by the Galactic tidal field.

Kim 2 lies at a heliocentric distance of $104.7 \pm 4.1 \mathrm{kpc}$. With a half-light radius of $12.8 \pm$ $0.6 \mathrm{pc}$ and ellipticity of $\epsilon=0.12 \pm 0.10$, the cluster features the properties of typical outer halo globular clusters, except for the rather high metallicity $\left([\mathrm{Fe} / \mathrm{H}]=-1.0_{-0.21}^{+0.18}\right)$ and unusually low luminosity $\left(M_{V}=-1.5 \pm 0.5\right)$. These parameters are similar to those for the outer halo globular cluster AM 4, which is thought to be associated with the Sagittarius dwarf spheroidal galaxy. In addition, the cluster exhibits evidence of significant mass loss 

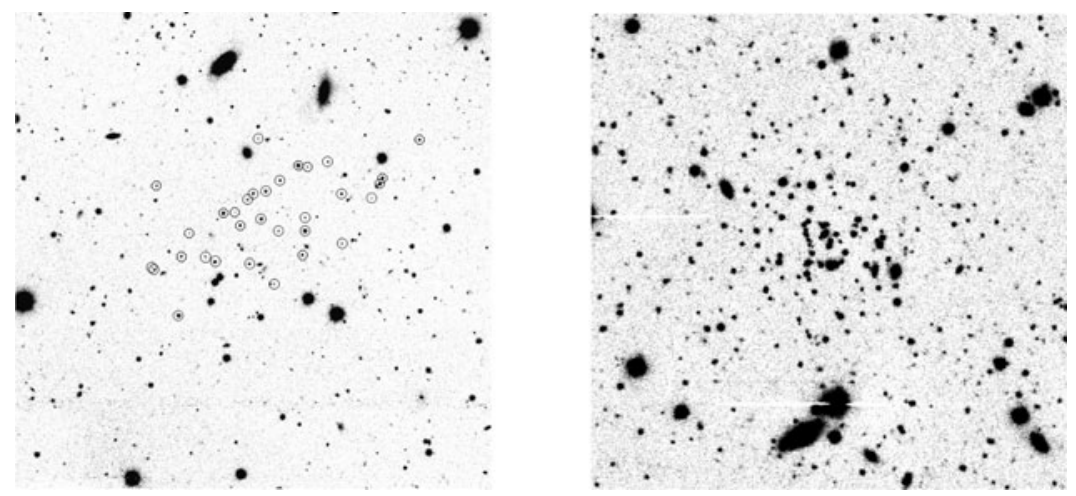

Figure 1. Left panel: $6 \times 6 \operatorname{arcmin}^{2}$ DECam cutout g-band image of Kim 1 (from Kim \& Jerjen 2015a). Member stars are highlighted with circles. Right panel: $4 \times 4 \operatorname{arcmin}^{2}$ GMOS cutout g-band image of Kim 2 (from Kim et al. 2015a). The cluster is located at the centre of the image. North is up, east is to the left.
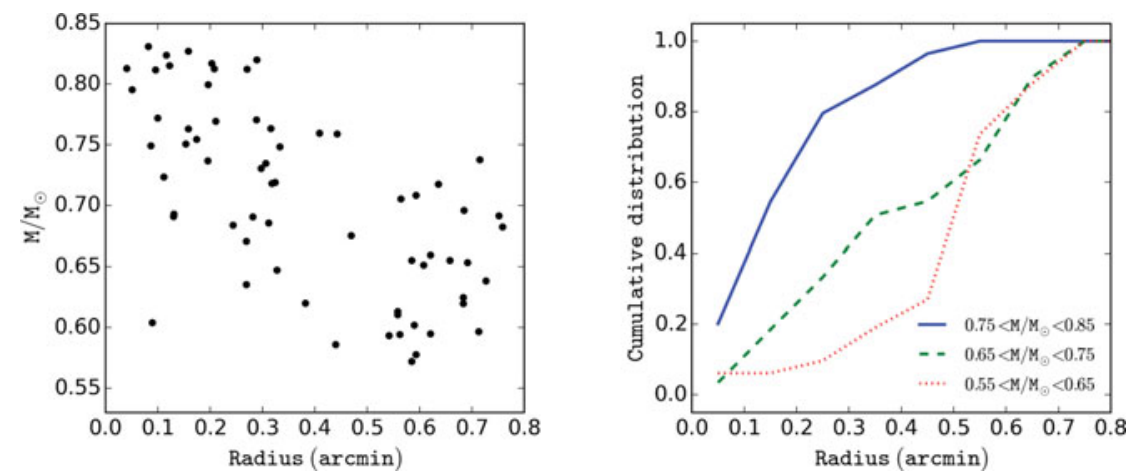

Figure 2. Mass segregation in Kim 2 (from Kim et al. 2015a). Left panel: stellar mass distribution of Kim 2 main sequence stars within $2 r_{h}(\sim 0.8 \mathrm{arcmin})$ as a function of the distance from the cluster center. Right panel: cumulative distribution function of Kim 2 main sequence stars for three different mass intervals.

such as extra-tidal stars and mass-segregation (Fig. 2). An outer halo globular cluster with such low star density is likely to follow an orbit confined to the peripheral region of the Milky Way halo, and/or to have formed in a dwarf galaxy that was later accreted into the halo (Sollima et al. 2011). Consequently, the cluster could have avoided major tidal disruption and survived until the present epoch.

\section{References}

Ahn, C. P., Alexandroff, R., Allende Prieto, C., et al. 2014, ApJS, 211, 17

Fadely, R., Willman, B., Geha, M., et al. 2011, AJ, 142, 88

Kim, D. \& Jerjen, H. 2015a, ApJ, 799, 73

Kim, D., Jerjen H., Milone, A. P., Mackey, D., \& Da Costa, G. S. 2015a, ApJ, 803, 63

Kim, D., Jerjen H., Mackey, D., Da Costa, G. S., \& Milone, A. P. 2015b, ApJL, 804, L44

Kim, D. \& Jerjen, H. 2015b, ApJL, 808, L39

Koposov, S., de Jong, J. T. A., Belokurov, V., et al. 2007, ApJ, 669, 337

Muñoz, R. R., Geha, M., Côté, P., et al. 2012, ApJL, 753, L15

Pawlowski, M.S., McGaugh, S.S., \& Jerjen, H. 2015 MNRAS, 453, 1047

Sollima, A., Martínez-Delgado, D., Valls-Gabaud, D., \& Peñarrubia, J. 2011, ApJ, 726, 47 